\title{
Neuronal Circuitry for Comparison of Timing in the Electrosensory Lateral Line Lobe of the African Wave-Type Electric Fish Gymnarchus niloticus
}

\author{
Masashi Kawasaki ${ }^{1,2}$ and Yuan-Xing Guo ${ }^{1}$ \\ 1University of Virginia, Department of Biology, Charlottesville, Virginia 22903 and 2 University of California at San Diego, \\ Scripps institution of Oceanography, San Diego, California 92093
}

An African wave-type electric fish, Gymnarchus, compares timing on the order of microseconds of sensory feedback from its high-frequency $(\sim 400 \mathrm{~Hz})$ electric organ discharges (EODs) received at different parts of its body surfaces. This capability is essential for and demonstrated by the jamming avoidance response (JAR). The organization of the timing comparison mechanisms was identified in the electrosensory lateral line lobe (ELL) in the hindbrain by field potential, extra- and intracellular recordings, and intracellular labeling with biotinylated agents.

Timing or phase of the EOD feedback is carried by action potentials of S-type primary afferent fibers that project to the inner cellular layer $(\mathrm{ICL})$ of the medial zone of the ELL and to the giant neurons in the ELL. The giant neurons bilaterally project to the ICL, where neurons sensitive to phase differences between different parts of the body occur. Although sensitive to dynamic phase changes of several microseconds, these differential- phase-sensitive neurons showed adaptation to steady-state changes of phase difference over a wide range (greater than $\pm 100 \mu \mathrm{sec}$ ) and continued to respond to small modulations after the mean difference was shifted.

Gymnarchus and an independently evolved South American electric fish, Eigenmannia, exhibit nearly identical JARs and share a rather complex but identical set of computational algorithms for JAR. This study showed that one of the computational steps, the timing comparison between body surfaces, occurs in the hindbrain in Gymnarchus, in contrast to the midbrain in Eigenmannia. Thus, similar systems with a similar overall function may have evolved differently in different genera by assigning a subfunction to different substructures within the brain.

Key words: electric fish; jamming avoidance response; phase comparison; binaural comparison; convergent evolution; adaptation
An African wave-type electric fish, Gymnarchus, and a South American wave-type electric fish, Eigenmannia, have evolved independently and are distantly related electric fishes that lack common electroreceptive ancestors (Lauder and Liem, 1983). Both fishes, however, perform a very similar jamming avoidance response (JAR) (Bullock et al., 1975) that requires a complex neural computation. In the JAR, the fish shifts the otherwise constant frequency (individually fixed at $250-500 \mathrm{~Hz}$ ) of its electric organ discharges (EODs) upward or downward away from the frequency of a neighbor's discharges to avoid mutual jamming of their electrolocation function.

A behavioral study on Gymnarchus discovered computational algorithms for the JAR that are remarkably complex and similar to those used by Eigenmannia. The shared features of neuronal computation in these species are as follows: (1) sole reliance on afferent information without internal reference to pacemaker; (2) decoding of the sign of frequency difference between the fish's and

Received July 12, 1995; revised Sept. 1, 1995; accepted Sept. 7, 1995.

The initial part of this study was aided by grants to Walter Heiligenberg from National Institutes of Health, the National Institute of Mental Health, and the National Science Foundation and from the Director's Office, Scripps Institution of Oceanography. We thank Dr. Theodore $H$. Bullock for his generous support for a part of this study at University of California at San Dicgo. This study was also supported by NIMH Grant R29 MH48115-01A1 to M.K. We thank Yasuko Kawasaki (University of Virginia) and Grace Kennedy (University of California, San Diego) for their excellent technical assistance. We thank two anonymous referees for critical comments and Cameron McLaughlin for editing English.

Correspondence should be addressed to Masashi Kawasaki, Department of Biology, Gilmer Hall, University of Virginia, Charlottesville, VA 22903.

Copyright (C) 1995 Society for Neuroscience $0270-6474 / 95 / 160380-12 \$ 05.00 / 0$ its neighbor's discharge from a temporal pattern of amplitude modulation and phase modulation in afferent signals; (3) differential-phase comparison; and (4) resolution of spatial ambiguity by spatial averaging (Kawasaki, 1993a). Since Gymnarchus and Eigenmannia have evolved electrosensory systems independently, their neuronal implementation of these computational steps is not necessarily expected to be identical. These two genera provide a rare opportunity to study complex vertebrate neuronal mechanisms that have cvolved independently to perform a similar task. Although the neuronal mechanisms for the JAR in Eigenmannia are well studied (Heiligenberg, 1991), nothing is known about neurophysiological mechanisms for the JAR in Gymnarchus except for its receptor physiology (Bullock et al., 1975).

Since Gymnarchus emits a wave-type EOD of constant frequency, the timing of zero-crossings, or phase, of electrosensory feedback of EODs at electroreceptors is constant when the animal's electroreceptors are stimulated solely by its own EOD. In a jamming situation, however, the electroreceptors are stimulated by the sum of the fish's and its neighbor's EODs, which modulates in phase as well as in amplitude at a frequency equal to the absolute frequency difference between the two fish.

Lacking an internal temporal reference, Gymnarchus cannot directly sense phase modulation, even though its time course is essential for the JAR. Rather, Gymnarchus extracts the time course of phase modulation from differences in magnitude of phase modulation between different locations on its body surface, which experience different amplitude ratios between the fish's own and its neighbor's EOD. Since the magnitude of phase 
modulation is a function of the amplitude ratio, different body surfaces experience phase modulation with different magnitudes. Experimental elimination of the phase difference abolishes JAR (see Figs. 2, 3, 8 in Kawasaki, 1993a). Local phase information must be conveyed to the central nervous system, and central neurons must compare phases between different body areas to extract the time course of the phase modulation.

This study identified the neuronal mechanism for the phase information processing. Phase comparison was found to occur in Gymnarchus in the electrosensory lateral line lobe (ELL) in the medulla - a different brain structure from the torus semicircularis in the midbrain used by Eigenmannia for the same task (Carr et al., 1986a,b).

\section{MATERIALS AND METHODS}

Approximately 60 Gymnarchus niloticus $(11-17 \mathrm{~cm})$ were used. Environmental conditions in holding tanks were identical to those described in Kawasaki (1994). Intramuscular injection of Flaxedil (gallaminc triethiodide, $0.03 \%, 3-8 \mu \mathrm{l}$ ) immobilized fish and attenuated EODs significantly. The immobilized preparation was also maintained by the methods described in Kawasaki (1994). After local application of Xylocane (2\%), a small hole was drilled above the corpus cerebelli. The caudal part of the ELL was exposed by removing the caudal edge of the corpus cerebelli with a fine suction tubing (inner diameter $500 \mu \mathrm{m}$ ). Field potential was recorded by glass capillary electrodes filled with $3 \mathrm{M} \mathrm{NaCl}$ (tip diameter $5 \mu \mathrm{m})$. Extracellular single-unit recordings were made by indium-filled glass capillary electrodes (Frank and Becker, 1964). Intracellular potentials were recorded by glass capillary electrode filled with $2 \%$ biocytin (Sigma) dissolved in $2 \mathrm{M}$ potassium acetate or $2 \%$ neurobiotin (Vector) in $2 \mathrm{M}$ potassium chloride. After recording, biocytin or neurobiotin was iontophoresed intracellularly by passing positive current $(+1.5 \mathrm{nA}$ offset, $3 \mathrm{nA}$ peak-to-peak, sinusoidally modulated at $2 \mathrm{IIz}$ ). Extracellular injection of neurobiotin was made by a glass capillary electrode (tip diameter $5 \mu \mathrm{m}$ ) filled with $2 \%$ neurobiotin in $2 \mathrm{M}$ potassium chloride for retrograde labeling. Positive DC current of $2 \mu \mathrm{A}$ was applied for $15 \mathrm{~min}$ to $1 \mathrm{hr}$. Survival time for the retrograde transport from the torus semicircularis to the ELL was $7 \mathrm{hr}$.

At the conclusion of experiments, the fish was anesthetized with MS222 (1:5000) and perfused through the heart with Hickman's Ringer followed by $4 \% p$-formaldehyde in $0.1 \mathrm{M}$ phosphate buffer, $\mathrm{pH} 7.4$. The brain was sectioned at $50 \mu \mathrm{m}$ on a vibratome. Biocytin and neurobiotin were visualized with the method after Horikawa and Armstrong (1988) as described in Kawasaki (1993a). Sections were counterstained with Neutral Red. Neurons were drawn with a drawing tube under a $100 \times$ or $40 \times$ objective lens.

The axons of giant neurons of ELL were counted in a Toluidine Blue-stained semithin section $(0.5 \mu \mathrm{m})$ sagittally cut along the midline in one fish $(13.5 \mathrm{~cm})$. The semithin section was prepared after embedding a vibratomed section in Eponate 812 (Ted Pella). Somata of giant neurons in the same fish were counted by reconstructing all somata from all sections that were heavily stained with Neutral Red.

Electrosensory stimuli were applied in the "phase-chamber" configuration described in Kawasaki (1993a). In this configuration, the body surface was isolated electrically into two portions (head and trunk compartments) with a partition between the eyes and the base of the pectoral fins that was tightly sealed to the fish's skin by means of silicone grease (High Vacuum Grease, Dow Corning) (Fig. 1A). The electrical isolation between the compartments was $>40 \mathrm{~dB}$. An unmodulated sinusoidal signal with a frequency near that of the fish's own pacemaker $(350-420$ $\mathrm{Hz}$ ) was applied in the trunk compartment between an electrode inserted in the mouth and a pair of electrodes straddling the trunk. The head compartment was stimulated by a phase-modulated sinusoidal signal with the same carrier frequency that was applied between a pin electrode inserted near the dorsal fin and a pair of electrodes straddling the head. The pin electrode was insulated electrically by paint except at its tip.

Phase of the head signal was finely controlled in reference to the sinusoidal signal in the trunk compartment. The phase of the head signal was modulated sinusoidally typically at $1 \mathrm{~Hz}$ (Fig. $1 B, C$ ). The magnitude of modulation was $\pm 80 \mu \mathrm{sec}$ for the search stimulus and could be reduced to $\pm 1 \mu \mathrm{sec}$. The center of the sinusoidal modulation (center phase) could be offset as desired. These signals were generated and controlled by a phase synthesizer (WT650, Wavetek) that generates two sinusoidal waves
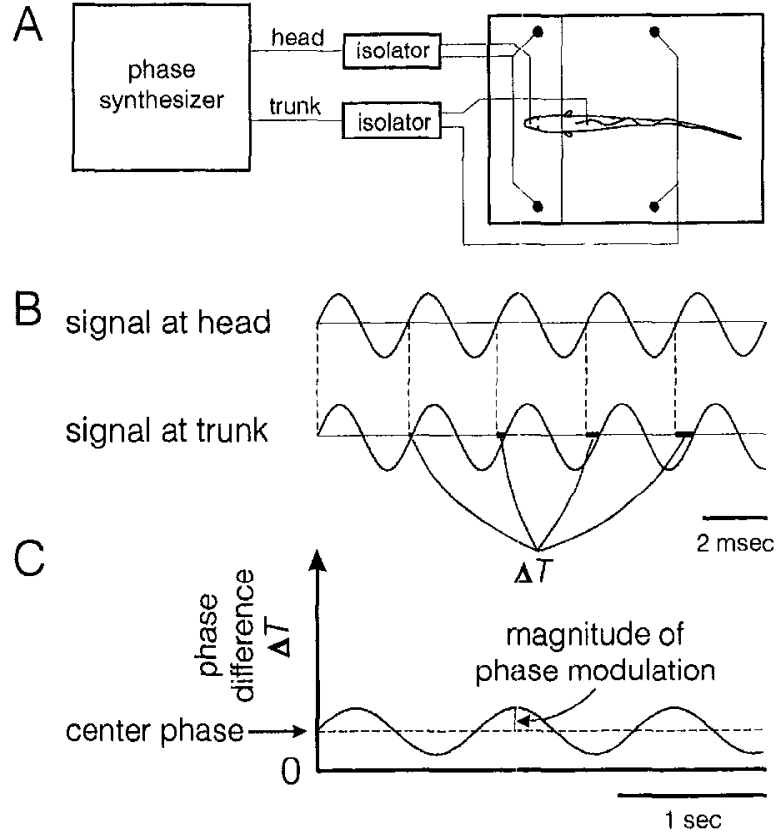

Figure 1. Electrosensory stimulus applied in the phase-chamber configuration. $A$, Fish's skin surface is electrically isolated into two parts, a head compartment and a trunk compartment. $B$, While the head compartment received an unmodulated sinusoidal signal, the sinusoidal signal in the trunk compartment was modulated in phase. The phase difference between the head and trunk signal is defined as $\Delta T, \Delta T<0$ and $\Delta T>0$ being delay and advance, respectively, of the head signal in reference to the trunk signal. $C$, Phase modulation function (1 Hz sine wave) was fed into the phase synthesizer to generate the differential phase modulation between the head and trunk compartments. The center phase and the magnitude of the modulation could be altered independently.

with precise phase relationship according to an externally applied signal. The phase accuracy of the signal generator was $\sim 1 \mu \mathrm{sec}$. For more accurate differential-phase presentation $(<1 \mu \mathrm{sec})$, the base frequency of the phase synthesizer was set at a frequency 1000 times higher than that of the normal signal. Its outputs were then fed into divide-by-1000 counters, creating two square waves at $\sim 400 \mathrm{~Hz}$, the phase accuracy of which was improved to $10 \mathrm{nsec}$. These square waves were applied to the two compartments, and phase of the head signal in reference to the trunk signal was controlled by an external command signal much as in the sinusoidal signal presentation. In all cases, the signal amplitude was set at $2-3 \mathrm{mV} / \mathrm{cm}$ measured at the skin surface. This value approximated the amplitude of natural EOD feedback.

Field potential and intracellular potentials were recorded by an electrometer (S7071A, WPI). Extracellular potentials were recorded by a homemade FET preamplifier. For extracellular recordings, the large field potential (up to $10 \mathrm{mV}$ ) caused by phase-locked firing of primary afferent terminals and giant neurons was eliminated by a cancellation technique in which the recorded signal was delayed by one signal cycle, inverted in sign, and added to its original version, canceling all constant signal components with fundamental and higher harmonics (Bastian and Heiligenberg, 1980).

Stimulus parameters and neuronal potentials were recorded by a tape recorder (TEAC-101 or HP3964A) and analyzed later by computers (PDP-11/73, IBM-P5, and IBM-486 equipped with a peripheral interface by 'l'ucker-Davis).

The degree of synchronization between stimulus sinusoids and action potentials of ELL neurons was measured as vector strength (Goldberg and Brown, 1969), computed as $\nu=\left[\left(\Sigma x_{i} \cdot \cos _{i} / n\right)^{2}+\left(\sum x_{i} \cdot \sin _{i} / n\right)^{2}\right]^{1 / 2}$, where $x_{i}$ is the height of the $i$ th bin in stimulus cycle histograms, $\cos _{i}=$ $\cos (i \cdot 2 \pi / n)$, and $\sin _{i}=\sin (i \cdot 2 \pi / n), i=1, \ldots, n, n$ being the total number of bins in a cycle histogram. The response magnitude of ELL neurons to sinusoidal phase modulation was measured by the fundamental Fourier component of response histogram in units of spikes $/ \mathrm{sec}$ plotted against phase modulation. This measure expresses the magni- 

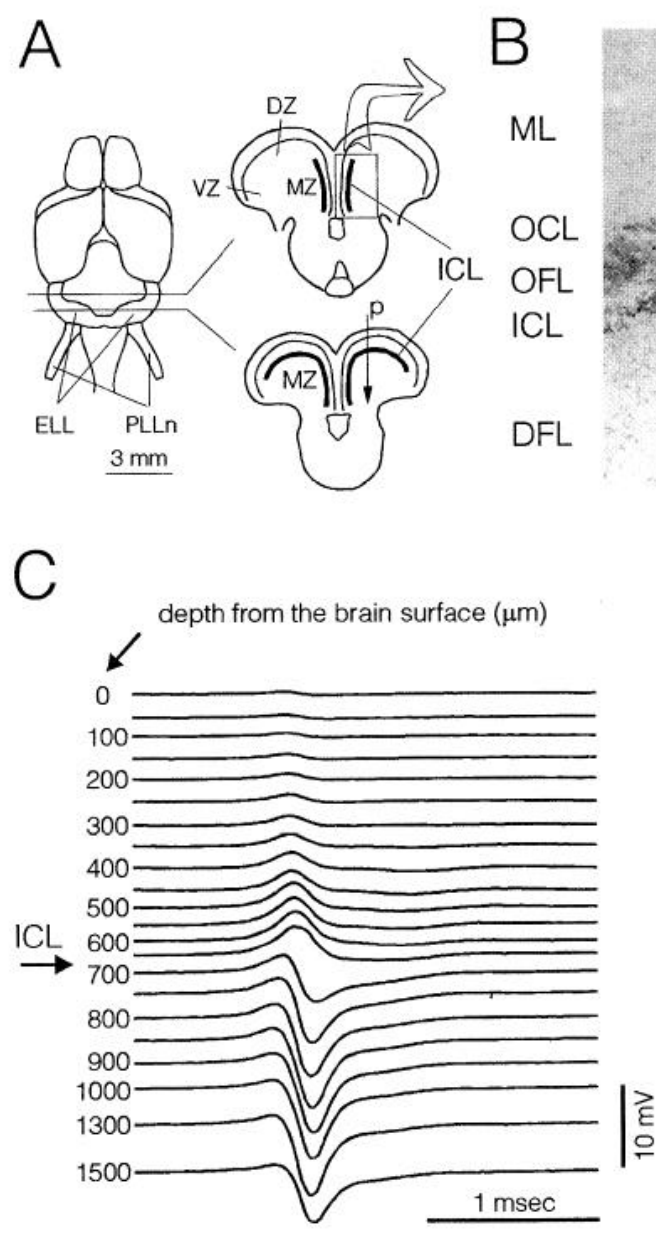

tudes of changes of spontaneous discharge frequency in response to phase modulation.

A neuron's threshold sensitivity to modulations of phase difference was determined by the sign test. The numbers of spikes in the first and the second halves within individual modulation cycles were compared, and significance was tested across modulation cycles. The number of modulation cycles tested was between 10 and 30 .

Anatomical nomenclature of brain structures of Gymnarchus follows Bass and Hopkins (1982) unless otherwise mentioned.

\section{RESULTS}

\section{The ELL}

The ELL of Gymnarchus consists of bilateral lobes at the caudal end of the brain, each comprising three distinctive zones: dorsal (DZ), ventral (VZ), and medial (MZ) (Bass and Hopkins, 1982). Figure 2 shows the ELL zones and their layers. A transverse section of the ELL at $\sim 800 \mu \mathrm{m}$ to the caudal end of the ELL contains a large part of the medial zone. As the medial zone extends rostrally, the lateral extent of the zone becomes smaller and the zone disappears at $\sim 1700 \mu \mathrm{m}$ from the caudal edge of the ELL. The medial zone consists of five layers: molecular (ML), outer cell (OCL), outer fiber (OFL), inner cell (ICL), and deep fiber (DFL). Throughout the medial zone, a large field potential that was phase-locked to the sinusoidal stimulus was recorded in the DFL (Fig. 2C). This large field potential was absent from any other zones and abruptly ended at the ICL, indicating that phaselocked inputs are provided by the DFL and terminate at the ICL. No sign of phase-locked field potential was detected in the torus semicircularis in the midbrain.

\section{Primary afferents}

Approximately 150 afferent fibers were intracellularly penetrated at the base of the posterior lateral line nerve. Four distinct response types were encountered: S-type, O-type, ampullary, and mechanosensitive. S-type fibers responded with an action potential for each cycle of the sinusoidal stimulus in a phase-locked manner over a wide range of stimulus amplitude. Stimulus intensity required for the one-to-one firing was $\sim 0.1 \mathrm{mV} / \mathrm{cm}, 20-30 \mathrm{~dB}$ lower than the natural range of stimulation by the fish's own EOD (Fig. $3 A$ ). These fibers showed characteristic ringing in the absence of stimulus at a frequency close to that of the fish's own pacemaker activity (Fig. $3 B$ ). This is due to ringing of electroreceptors. O-type fibers fired an action potential every several cycles of the sinusoidal stimulus, and the probability of firing was a function of stimulus intensity. Unnaturally large stimulus intensities $(\sim 15 \mathrm{mV} / \mathrm{cm})$ were necessary to drive O-type fibers one-to-one to every stimulus cycle. These fibers were usually silent in the absence of stimulus (Fig. $3 C, D$ ). Ampullary fibers showed spontaneous discharges $(\sim 70 \mathrm{~Hz})$, and their firing rate was modulated by an sinusoidal stimulus of low frequencies $(<100 \mathrm{~Hz})$ (Fig. $3 E$ ). Ampullary responses have been recorded in the lateral line nerve in Gymnarchus for the first time in this study. While lowfrequency information is not required for the JAR, ampullary receptors are suited to detect low frequency components in the EOD interruption behavior of Gymnarchus, which is exhibited in their communication (Hopkins, 1974). Mechanosensitive fibers were indifferent to any electrical stimulation tested but vigorously responded to manually induced ripples in the experimental tank (not shown). 


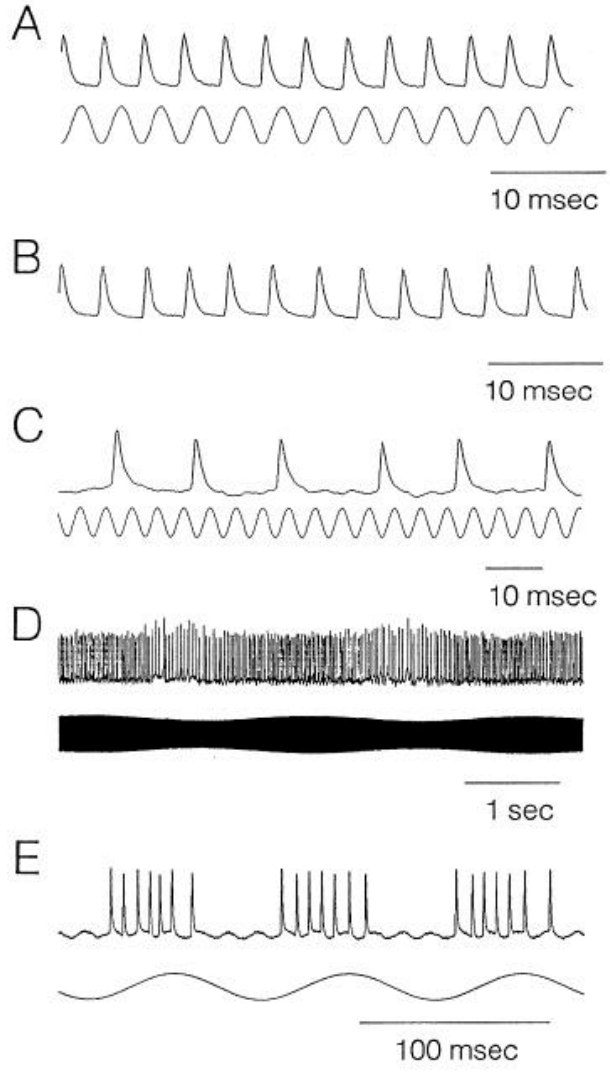

Figure 3. Physiological responses of primary afferent fibers intracellularly recorded at the proximal end of the posterior lateral line nerve. $A$, An $\mathrm{S}$-type afferent. Note one-to-one firing to stimulus cycles. $B$, Ringing of the same fiber without stimulation. $C$, An O-type afferent. $D$, The same recording with longer time scale showing a varied spiking rate in response to amplitude modulation. $E$, An ampullary afferent showing burst responses to a $10 \mathrm{~Hz}$ signal.

Intracellular labeling of S-type fibers revealed two morphological types. The afferent fibers of the first type are $\sim 3 \mu \mathrm{m}$ in diameter because they run in the proximal portion of the posterior lateral line nerve. After the afferent fibers enter the brain, their diameter increases to $5 \mu \mathrm{m}$ and they give off several fine branches (1.5 $\mu \mathrm{m}$ in diameter), which may ramify further into many terminal fibers that form synaptic contacts onto the soma of one to several giant neurons. The main axon keeps its thickness and continues to run within the DFL toward the ICL of the medial zone. Before reaching the ICL, it ramifies into glomerular terminals that end at the ICL (Fig. 4) but never penetrate beyond it. The second type of the S-type afferents (not shown) is indistinguishable from the first type, except that they lack the axon collaterals projecting to the giant neurons. Their sole connection is to the ICL. A few O-type fibers were labeled. None terminated onto the giant neurons or the ICL but, rather, projected to the dorsal zone of the ELL.

\section{Giant neurons}

The somata of giant neurons are large (38-45 $\mu \mathrm{m}$ in diameter) and were readily observable in Neutral Red-stained sections in the ventrolateral part of the DFL, dispersed over a wide range ( $\sim 1000 \mu \mathrm{m}$ rostrocaudally, $\sim 800 \mu \mathrm{m}$ laterally), with $\sim 130$ somata on one side. Intracellular recording from giant neurons showed phase-locked potentials similar to those of S-type afferent fibers. Intracellular labeling revealed their morphology (Fig. 5): the initial segment arises from the soma gradually and exhibits the minimum thickness $(\sim 1.5 \mu \mathrm{m})$ at $\sim 20 \mu \mathrm{m}$ from it. There the axon abruptly thickens, presumably because of myelination, to $10 \mu \mathrm{m}$ in diameter and runs medially for a few hundred micrometers. The axon then divides into the ipsilateral and contralateral branches (both $10-12 \mu \mathrm{m}$ in diameter). As the ipsilateral branch runs through the DFL, it gives off numerous finer branches. The bifurcated axon repeatedly branches into terminal fibers that terminate at the ICL. The contralateral axon branch runs across the midline and branches into an arborization before it reaches the contralateral DFL, much as the ipsilateral branch does. The terminals often show characteristic swelling at the ICL (Fig. $5 F, G)$. Each giant neuron had $\sim 20$ terminals on either side of the ELL and distributed its terminals over wide areas in the ICL. The commissural axons form a tight bundle, the diameter of which is $\sim 250 \mu \mathrm{m}$ at the midline. In one fish (13.5 $\mathrm{cm}$ in length), the numbers of somata of giant neurons on each side and the numbers of the commissural axons were counted as 143 (somata on left side), 125 (somata on right side), and 276 (axons). Thus, all giant neurons are presumed to have axonal branches on both sides of the ELL. In some cases where the soma was strongly labeled with biocytin, tcrminals of afferent fibers were labeled transsynaptically (Fig. 5D).

\section{Conduction velocity in the axon of giant neurons}

Conduction velocity at the axons of giant neurons was estimated by recording field potentials in the commissural tract of giant neurons. The fish's left and right sides were simultaneously stimulated by separate sinusoidal signals whose frequencies were identical but whose phases were $180^{\circ}$ apart, thus separating an otherwise single component of the field potential within the commissural tract into two components that could be ascribed to the activities of the axons of left and right giant neurons, respectively (Fig. 6). As the recording location of the field potential was moved away from the midline, one of those components was delayed and the other was advanced. Conduction velocity was calculated from the slope of the location-dependent latency shifts as $36 \mathrm{~m} / \mathrm{sec}$.

\section{Extracellular recording from differential-phase- sensitive neurons in the ELL}

The termination of phase-locked inputs, S-type afferents, and giant neurons at the ICL suggested that sensitivity to phase differences between these inputs emerge at this location. Extracellular recordings were made near the ICL in search of neurons that respond to phase differences between stimuli at the head and trunk. Recording electrodes were guided to the depth of the ICL in the MZ by the pattern of the depth-dependent change of field potential (Fig. $2 C$ ). In this area, we recorded from neurons $(n=$ 41 ), the firing rates of which were modulated in response to the phase differences between stimuli at the head and trunk. As shown in Figure 7, these units showed spontaneous discharges at $\sim 10-50$ spikes/sec, which was enhanced or suppressed according to the sign of phase difference between the compartments when phase difference was modulated at $1 \mathrm{~Hz}$. When we swapped the stimuli at the head and trunk, the response histograms were shifted by $180^{\circ}$ in the modulation cycle, further confirming that these neurons responded to phase differences (Fig. $7 C$ ). These neurons did not respond at all when we modulated absolute phase of signals in both compartments together with the same magnitude of modulation, indicating again that these neurons respond to phase differences but not to absolute phase modulation or to the resultant frequency modulation in either compartment (Fig. 7B). Ac- 
A
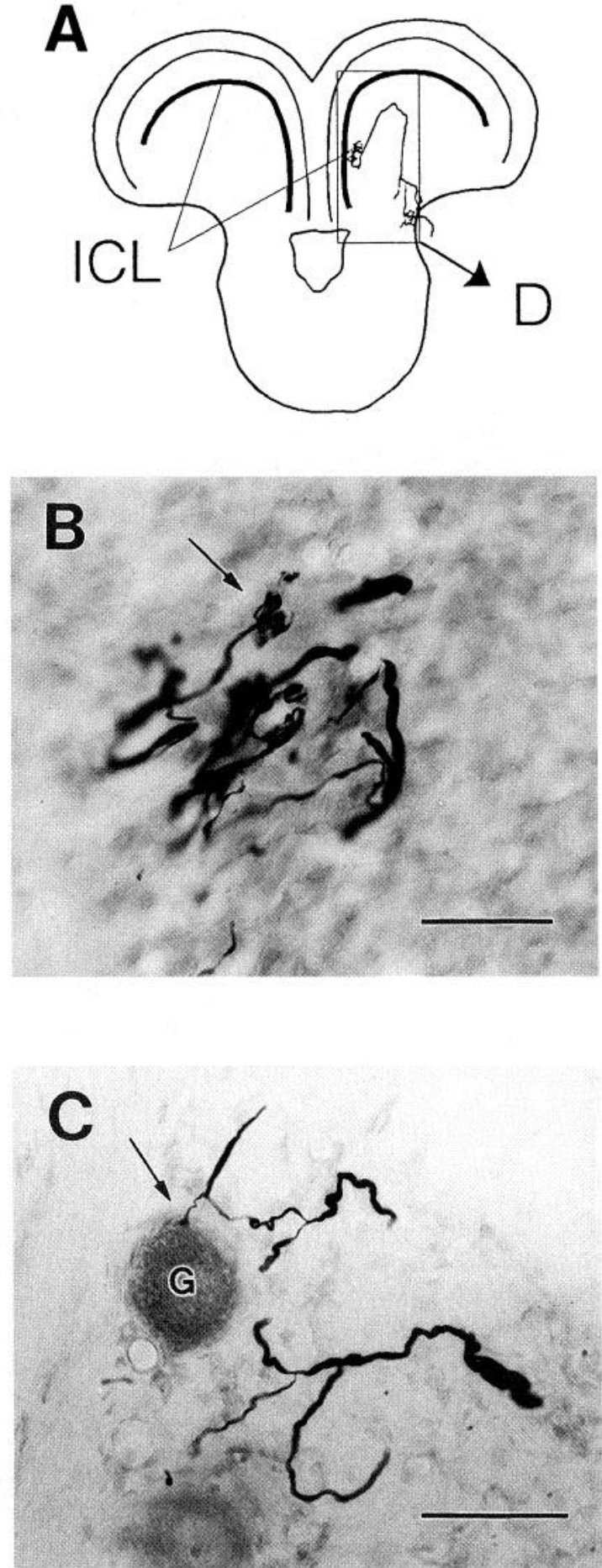

D

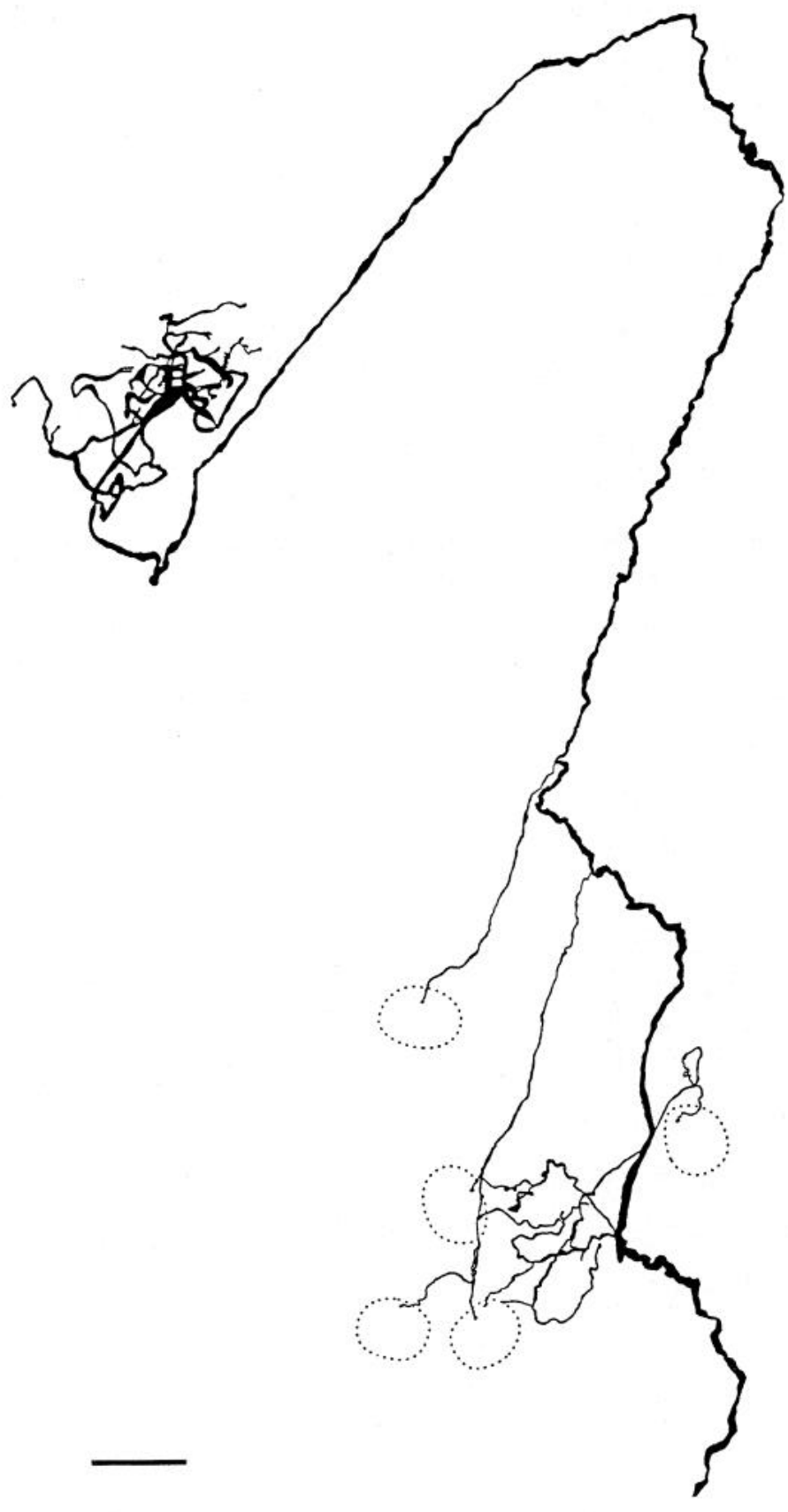

Figure 4. An intracellularly labeled S-type afferent fiber. $A$, Transverse section of the ELL showing the location of the terminal fibers in $D$. B, Photomicrograph of an S-fiber terminal (section thickness $50 \mu \mathrm{m})$ in the ICL. Notice a characteristic terminal morphology (arrow). $C$, A terminal (arrow) on the soma $(G)$ of a giant neuron. D, Camera Lucida drawing of an S-type afferent. Dotted circles represent somata of giant neurons contacted by this afferent fiber. Calibration bars, $50 \mu \mathrm{m}$.

Figure 5. Morphology of giant neuron. $A$, Camera Lucida drawing of a biocytin-filled giant neuron. Drawings from 15 transverse sections (50 $\mu \mathrm{m}$ thick) were reconstructed. Inset, A transverse section through ELL showing the position of the giant neuron. $B$, Photomicrograph of the ventrolateral end of the ELL showing somata of giant neurons stained with Neutral Red and the soma and axon of a biocytin-filled giant neuron. Scale bar, $200 \mu \mathrm{m}$. $C$, Highmagnification photograph of $B$ showing the initial segment. $D$, A trans-synaptically labeled afferent terminal fiber (arrow) onto the soma of a biocytin-filled giant neuron. $C$ and $D$ are from the same soma cut into two sections. $E$, The parent axon $(p)$ branches into the ipsi $(i)$ - and contralateral $(c)$ axons. $F$, $G$, Terminals of a biocytin-filled giant neuron at the ICL. Note the bulbar terminals anchored in the ICL. Calibration bars in $C-G$ are $50 \mu \mathrm{m}$. 
A
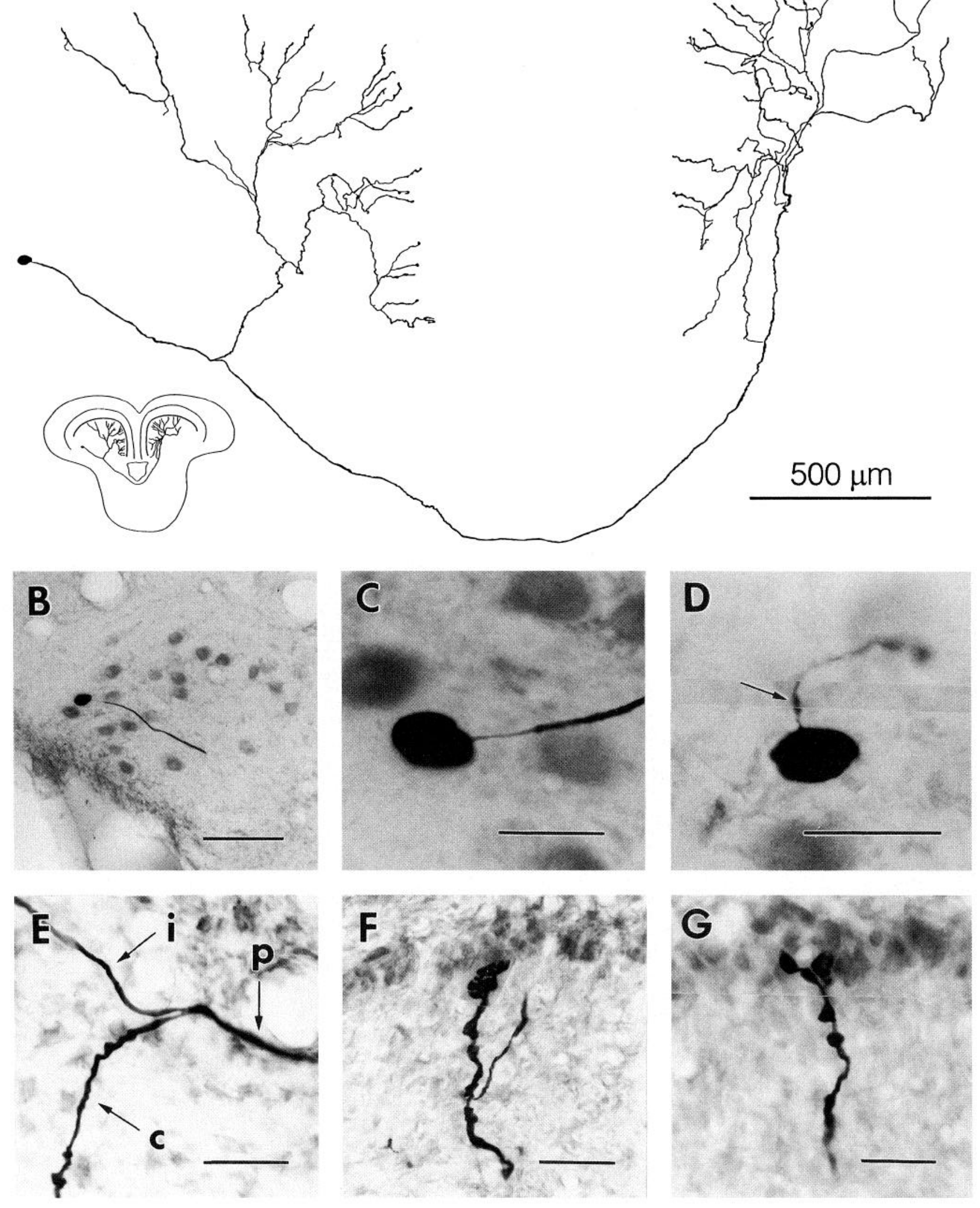
tion potentials from these neurons occur $\sim 1.0-1.4 \mathrm{msec}$ after the positive peak of the local field potential (Fig. 8).

Since firing times of phase-locked inputs to the differential-phasesensitive neurons from different body areas are expected to differ because of natural variations in conduction time from electroreceptors to these neurons, we investigated response properties of these neurons by varying the center value of sinusoidal phase modulation in the phase chamber. While modulating phase differences at $1 \mathrm{~Hz}$ in a sinusoidal manner $( \pm 20 \mu \mathrm{sec})$, we shifted the center value in stepwise manner (Fig. 9). When we shifted the center phase by 80 $\mu$ sec to the direction that decreased the firing rate of the ncuron in the phase modulation, the spiking was initially suppressed. After a few seconds, however, the responses to phase modulation recovered (Fig. 9A). Similarly, when we shifted the center phase in the opposite direction by $80 \mu \mathrm{sec}$, it initially gave a burst of spikes during which no distinctive responses to phase modulation were observed. After a few seconds, again, the overall firing rate decreased and the responses to pliase modulation reappeared (Fig. 9B). The adaplation to a new center value occurred within a limit of approximately a few hundred microseconds. If the center values were shifted beyond the limit, the neurons showed higher or lower spontaneous discharges depending on the direction of the shift and did not respond to phase modulations (Fig. 9C,D). Thus, these neurons responded differently to dynamic and static phase changes. Figure 10 compares the static and dynamic responses of the differential-phase-sensitive neurons. Dynamic responses were always much stronger than predicted from the static response curve. Ninctecn other ncurons tested in this manner showed similar results. Field potential and intracellular recording from giant neurons and S-type afferents showed that their timing of firing faithfully followed the steady-state change in stimulus phase, suggesting that the adaptation occurs within the ELL.

As the magnitude of phase modulation was diminished to below a few microseconds, peak and trough in the response histogram became less evident. Nevertheless, spike counts in the first and second halves in the modulation cycle histograms differed significantly, even with phase modulation of a few microseconds. Of 10 neurons tested in this manner, 7 neurons showed a significant preference to one sign of phase difference over the other when the magnitude of phase modulation was diminished to $<3.5 \mu \mathrm{sec}(p<0.01$, sign test). When the magnitude of phase modulation was reduced further to $1.7 \mu \mathrm{sec}, 4$ out of 10 neurons showed significant preferences $(p<0.01$, sign test). The most sensitive neuron differentiated phase modulation of $\pm 0.35 \mu \mathrm{sec}$ ( $p<0.01$, sign test) with the square wave stimulation (see Materials and Methods).

\section{Intracellular recording and labeling of differential- phase-sensitive neurons}

Intracellular recording was made in the medial zone from $>50$ neurons that showed sensitivity to phase difference. Morphology of 15 neurons was revealed by intracellular injection of biocytin or neurobiotin. Stable penetration of these neurons was difficult, and a recording with a $-50 \mathrm{mV}$ resting potential and $50 \mathrm{mV}$ action potentials was made in only one case. In other cases, action potential height remained $10-20 \mathrm{mV}$. Nevertheless, neurons were labeled successfully (Fig. 11).

Two morphological types of neurons showed differential-phase responses similar to those shown in the extracellular recordings. The first type of neuron had its somata in the ICL, and the other type had its soma in the OCL. The soma size of both types is $\sim 20$ $\mu \mathrm{m}$, with long apical spiny dendrites extending into the ML and basal dendrites spreading $\sim 100 \mu \mathrm{m}$. One ICL and one OCL neuron were strongly labeled and allowed us to track their axons, which projected to the torus semicircularis in the midbrain. Action potentials from both types of neurons were phase-locked well, but the degree of phase locking ("vector strength") was higher in neurons in the ICL $(0.97 \pm 0.025, n=7)$ and lower in neurons in the OCL $(0.61 \pm 0.21, n=10)$.

\section{Retrograde labeling of differential-phase-sensitive neurons from the torus semicircularis}

Extracellular injection of neurobiotin into the torus semicircularis retrogradely labeled ncurons in the ICL and OCL, the morphology of which was similar to intracellularly labeled differentialphase-sensitive neurons, having basilar dendrites and a thick apical dendrite that branched into spiny arborization in the ML of the medial zone (Fig. $2 B$ ).

\section{Response of differential-phase-sensitive neurons to amplitude modulation}

Although the primary cue for the differential-phase-sensitive neurons appears to be phase difference, these neurons do respond to amplitude modulations as well. Figure 12 shows the responses of differential-phase-sensitive neurons to amplitude-modulated signals. Firing times of intracellularly recorded action potentials of giant neurons' fibers were strongly dependent on the stimulus amplitude (Fig. 12G). This amplitude-dependent latency shift is responsible for the response of differential-phase-sensitive neurons to amplitude modulation.

\section{DISCUSSION}

\section{Phase comparison circuit in the ELL}

This study revealed the organization of the neuronal hardware for detecting phase differences-one of the essential computational elements for the JARs-in the ELL of Gymnarchus. Phase-locked action potentials from electroreceptors are supplied ipsilaterally by S-type afferent fibers and bilaterally by terminal axons of giant neurons to the ICL in the ELL, where neurons sensitive to phase differences emerge. The experiment in Figure 7 shows that differential-phase-sensitive neurons do respond to the difference in phase modulation between different body areas and do not respond to absolute phase modulation. Correspondingly, Gymnarchus does not use absolute phase information for its JAR (see Fig. 8 in Kawasaki, 1993a).

Whether the ICL neurons have direct contact with the terminals of giant neurons and S-type primary afferents remains to be examined by further studies involving electron microscopy of labeled neurons. The anatomical correspondence, observed at the light microscopic level, between the basilar dendrites of the ICL neurons and the terminals of phase-locked fibers, however, does suggest that the phase comparison takes place within the ICL. Differential-phase-sensitive neurons in the OCL do not appear to receive direct inputs from phase-locked elements, because their dendrites and the terminals of phase-locked neurons do not occur at the same level of the layers of the medial zone. OCL neurons are presumed to receive inputs from ICL neurons. The larger "vector strength" for the OCL than for the ICL neurons also supports this notion.

A relatively long firing latency of the differential-phase-sensitive neurons from the local field potential (1.1-1.4 msec; see Fig. 8) suggests chemical transmission between the phase-locked inputs and the differential-phase-sensitive neurons. While electrical transmission was found in the torus of Eigenmannia for phase comparison (Carr et al., 1986a), chemical transmission is also 
A
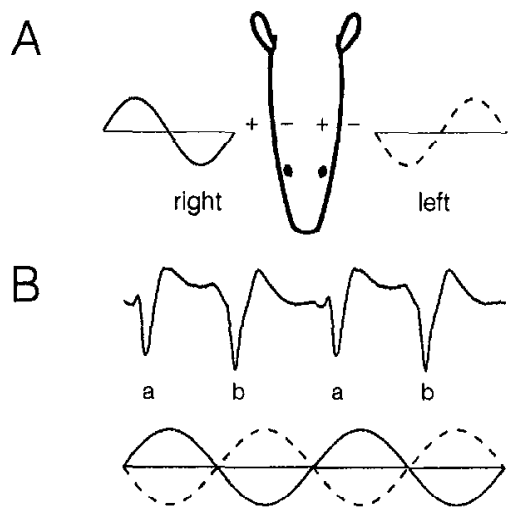

C

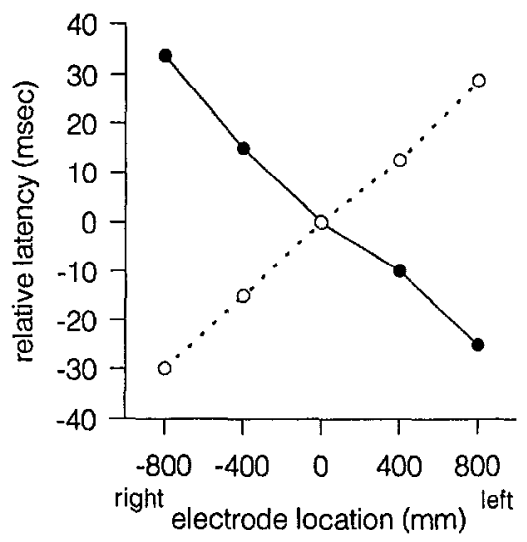

Figure 6. Estimation of conduction velocity of axons of giant neurons. $A$, Fish's left and right side are stimulated with sinusoidal signals of opposite phases. $B$, Field potential recorded within the commissural tract of giant neurons (top) showed two peaks ( $a$ and $b$ ) within a cycle of the stimulus sinusoid (bottom). The peaks $a$ and $b$ are ascribable to responses to the solid and broken sinusoids, respectively, because the peak $b$ merged into $a$ when the left-side signal (- - - -) was made identical to the right-side signal (-). $C$, Recording-site-dependent latency shift of the peaks $a$ and $b$. Average slope of these lines is $36 \mathrm{~m} / \mathrm{sec}$.

suggested in the nucleus of laminaris of the barn owl where interaural phase comparison occurs in the microsecond range (Konishi et al., 1988; Carr and Boudreau, 1993).

Of 10 differential-phase-sensitive neurons tested with small phase differences, 7 exhibited statistically significant responses to phase modulation of $<3.5 \mu$ sec. When phase difference was decreased further and the square wave was used as the carrier signal of the stimulus, one neuron showed significant responses to phase modulation of $0.35 \mu \mathrm{sec}$. Although a stimulation with a square-wave signal was not used in previous behavioral experiments and would decrease unnaturally the phase instability of phase-locked input to the ELL, this value indicates the performance limit of a single neuron in phase comparison within the ELL. These results represent the highest degree of neuronal sensitivity to small temporal differences so far found among other systems (Moiseff and Konishi, 1981; Harnischfeger et al., 1985; Carr et al., 1986a; Rose and Heiligenberg, 1986; Kawasaki et al., 1988; Kawasaki, 1993b).

The adendritic soma of giant neurons with a long and thin initial segment is another example of preserved or improved phase locking in neurons. The functional significance of a thin and long initial segment of the spherical cells in phase coding was pointed out first by Maler et al. (1981) and later was supported

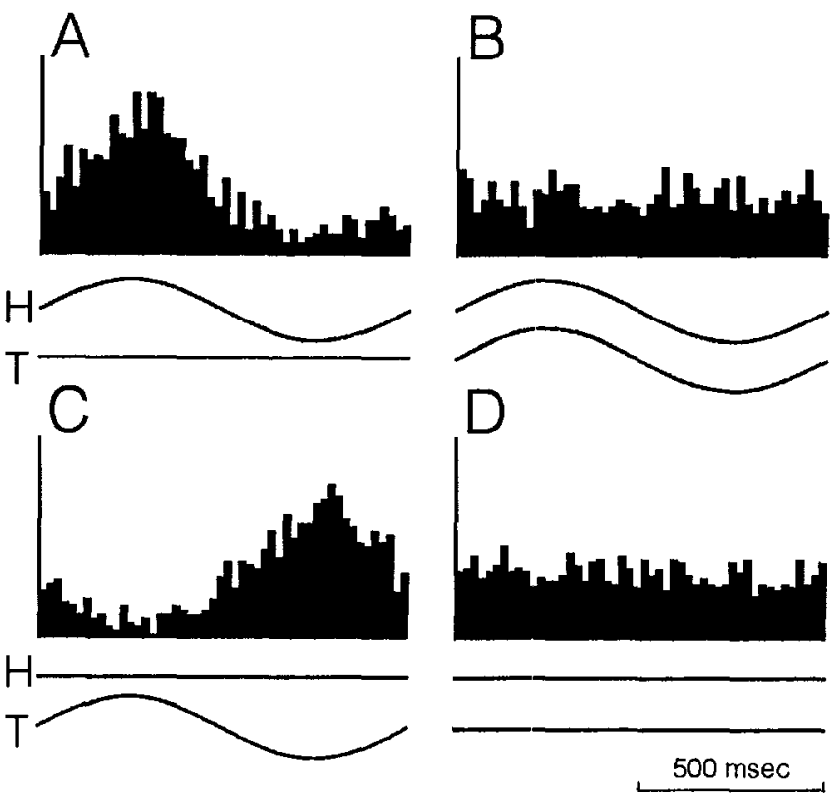

Figure 7. Spike histograms showing sensitivity of an ELL neuron to phase differences. Two traces below each histogram show absolute phase of signal at head $(H)$ and at trunk $(T)$. Phase advance and delay are plotted upward and downward, respectively. $A$, 'Irunk was stimulated with unmodulated sinusoidal signal; head was stimulated with a sinusoidal signal, the carrier frequency of which was identical but modulated in phase. The phase modulation was $\pm 70 \mu \mathrm{sec}$ at $1 \mathrm{~Hz}$. The neuron responds strongly when phase at head advances in reference to phase at trunk. $B$, Head and trunk received identical stimuli that modulated in phase as in head in $A$. $C$, Stimuli in $A$ were swapped between head and trunk. The neuron responds when phase at trunk delays, instead. $D$, Both head and trunk received unmodulated sinusoidal signal. Note that $B$ and $D$ are similarly indifferent. Bin width, $20 \mathrm{msec}$. Full vertical scale is 50 spikes $/ \mathrm{sec}$.

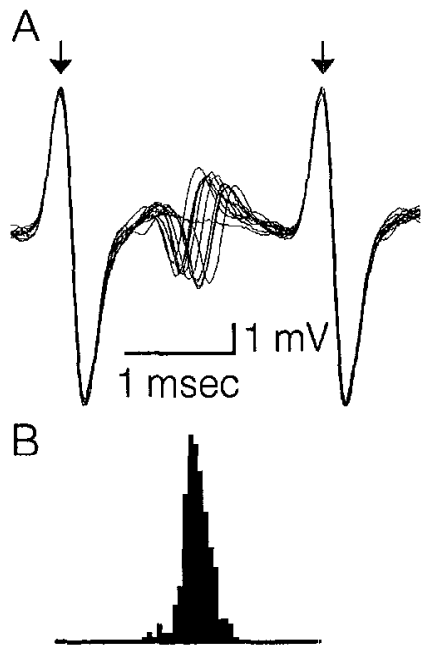

Figure 8. Timing of action potentials extracellularly recorded from a differential-phase-sensitive neuron. $A$, Spontaneously active spikes were recorded without differential phase modulation. Two large peaks (arrows) are field potential from phase-locked input to the ICL. Note similar waveform of field potential as in Figure $2 C$, indicating that recording was made near the ICL. Spiking rate was $\sim 10 / \mathrm{sec}$. Ten selected traces with a spike and one trace without a spike are superimposed. Note a latency of $\sim 1 \mathrm{msec}$ from field potential to spiking, $B$, Spike histogram plotted with the same time scale as in $A$, showing a high degree of synchronization of spikes to stimulus cycle. Vector strength of this units was 0.94 . 

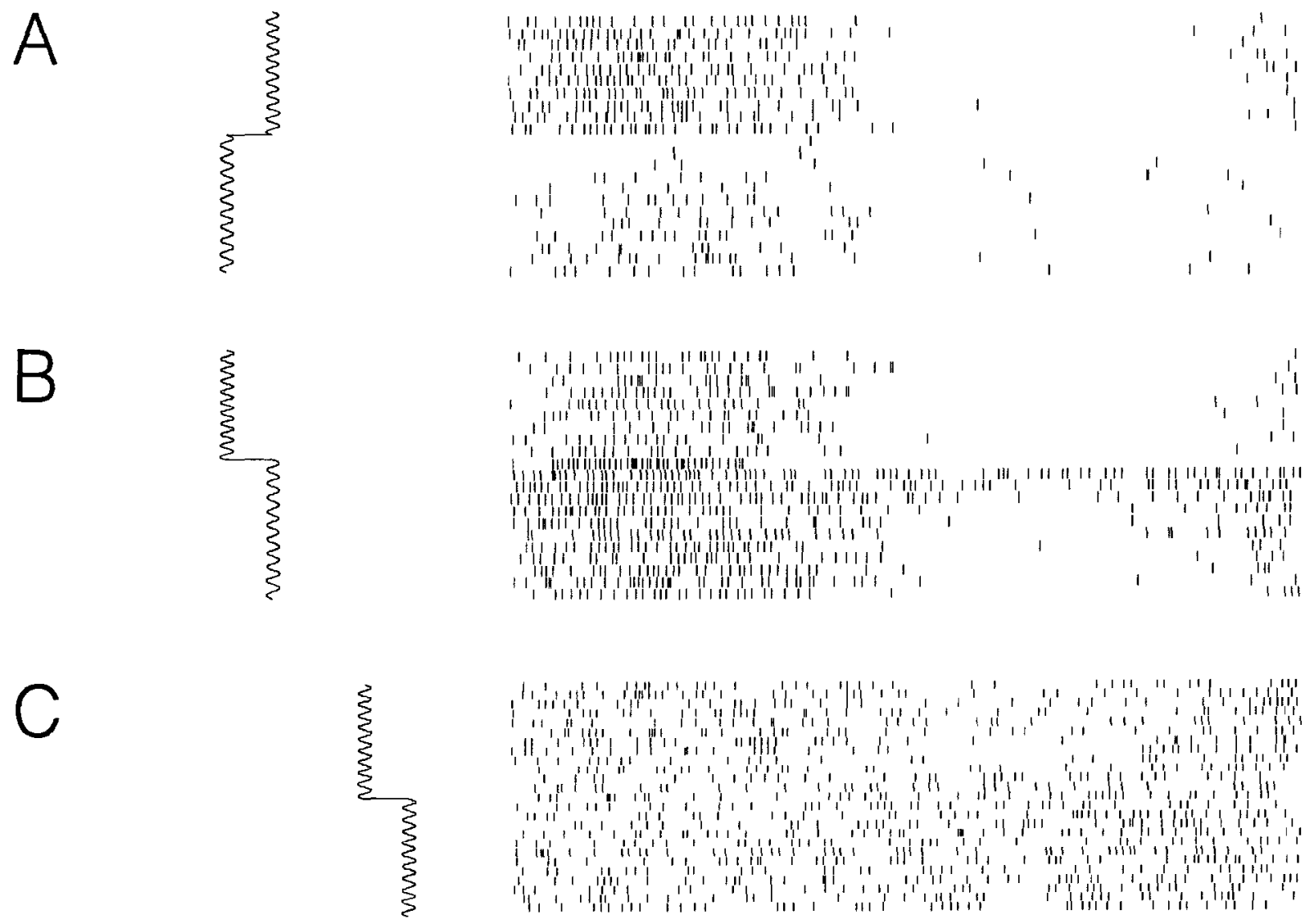

$0 \sum_{\xi}^{\xi}$
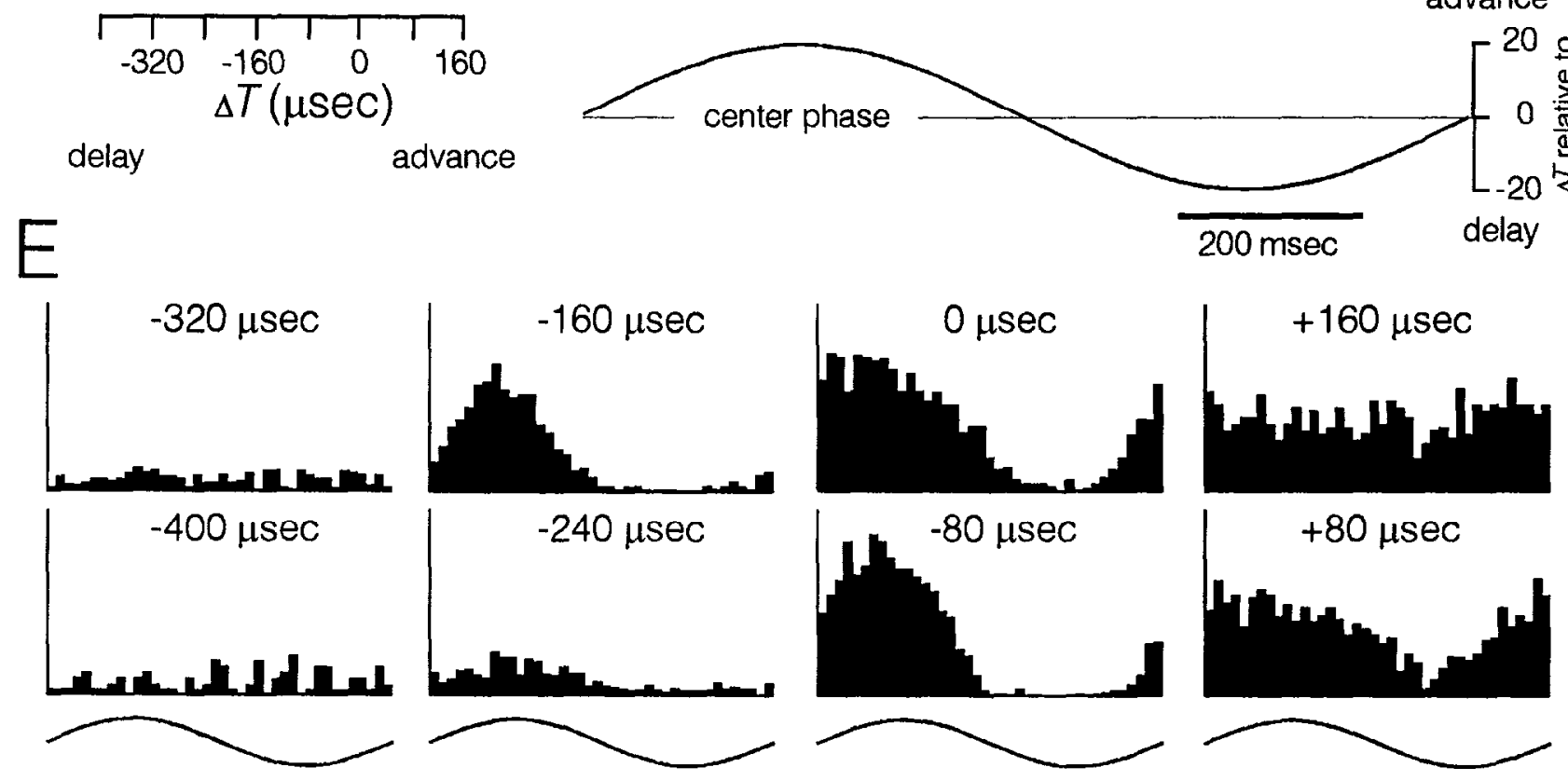


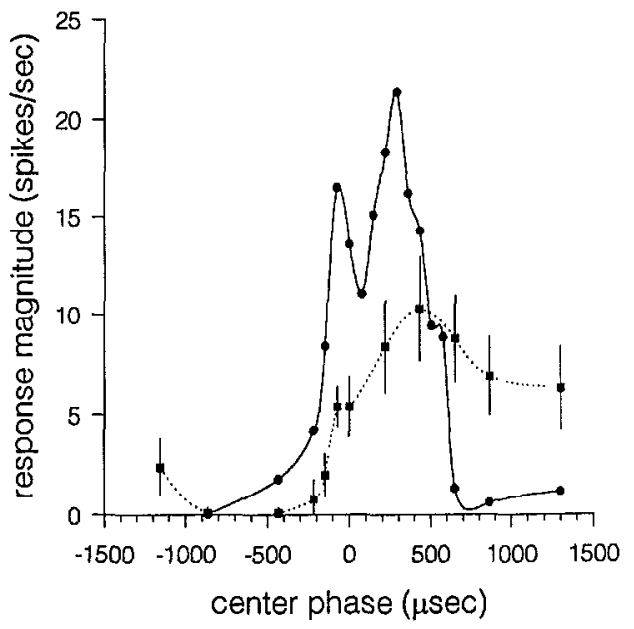

Figure 10. Comparison of responses of a differential-phase-sensitive neuron to steady-state and dynamic changes of phase difference. Firing rate was measured without sinusoidal phase modulation after $30 \mathrm{sec}$ of adaptation period at each center phase (steady-state responses, $\cdots$. ). Response magnitudes to sinusoidally modulating phase $( \pm 35 \mu \mathrm{sec}$ at $1 \mathrm{~Hz}$, see Materials and Methods) at various center phases are plotted in the same scale (dynamic response, - ).

physiologically by Carr et al. (1986a) in Eigenmannia. It is suggested that the degree of phase locking is improved by rejecting synaptic current from desynchronized inputs by the thin initial segment with high resistance. Similar phase coding and electromotor neurons are found in mormyrid electric fish [neurons in nELL (Bell et al., 1983) and in the lateral relay nucleus in Gymnarchus (Kawasaki, 1994)]. Presence of electrical synapses onto adendritic soma, which seems to be a general feature in these neurons, was suggested by transsynaptic labeling of input afferent from the giant neurons (Fig. 5D).

Intracellular labeling and retrograde transport showed that the differential-phase-sensitive neurons in the ELL project to the torus in the midbrain. Integration of differential phase and amplitude information-the next computational step for the JARmay occur, therefore, in the torus.

\section{Adaptation of differential-phase-sensitive neurons}

The differential-phase-sensitive neurons showed a large change of their firing rate in response to a dynamic phase modulation of small magnitude-a few microseconds in some cases. Yet when a large steady-state change of the center of the phase modulation was introduced, the neurons adapted to the new center phase and regained their responsiveness to small modulation after an initial loss of dynamic responses (Fig. 9A,B). The experiments in Figure 10 show that the responsiveness to dynamic, but small, changes in phase difference is preserved over a wide range of steady-state phase differences. Local mean amplitude of EOD feedback is subject to change due to the fish's stationary position against large

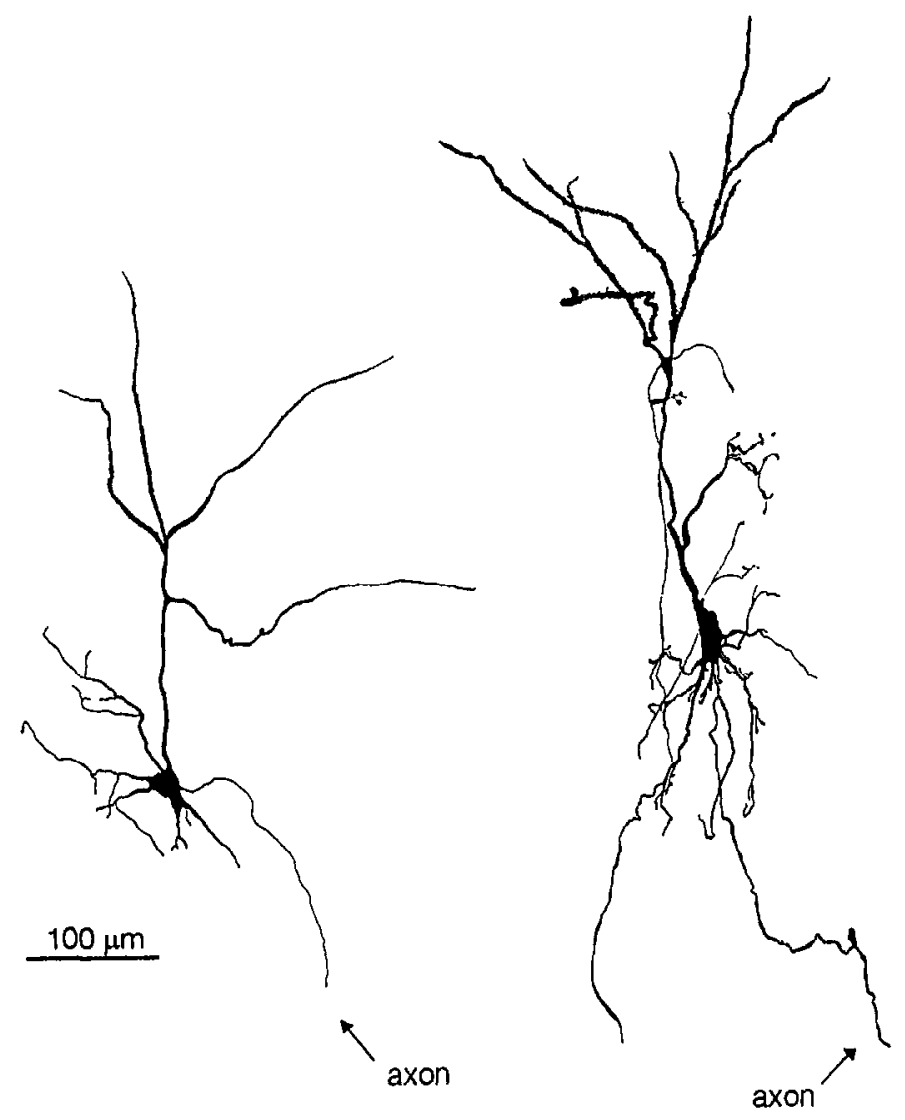

Figure 11. Camera Lucida drawing of differential-phase-sensitive neurons intracellularly labeled in the ELL. Left, The soma of this neuron resides in the ICL. A thick apical dendrite penetrates through the IFL and OCL, then ramifies into four branches within the ML. A similar apical dendrite is seen in Figure 2B. Finer basal dendrites spread within the ICL. Right, The soma of this neuron resides in the OCL where it spreads fine dendrites. Its apical dendrites also spread in the ML. No spatial relation was implied between the drawings of the two neurons. These neurons were located at the caudal end of the ELL.

objects or a hole in the natural environment. Such amplitude changes would cause stationary phase differences as predicted by Figure $12 G$. The ability of differential-phase-sensitive neurons to readjust to a new center phase may help the fish respond to dynamic changes in such circumstances.

Although the high conduction velocity $(36 \mathrm{~m} / \mathrm{sec})$ of the axon of the giant neurons reduces the conduction time loss in the commissural path between two halves of the ELL, it is still estimated to take as much as $\sim 70 \mu \mathrm{sec}$ for contralateral conduction. The timing mismatch between phase-locked signals from ipsi- and contralateral sides would also be nullified by this adaptation mechanism.

As shown in Figures 9 and 10, the adaptation is incomplete in

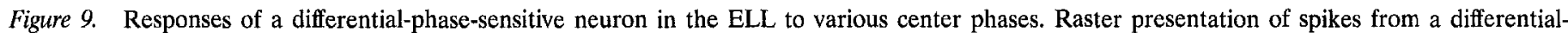

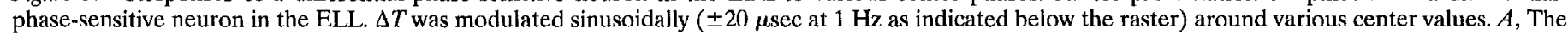

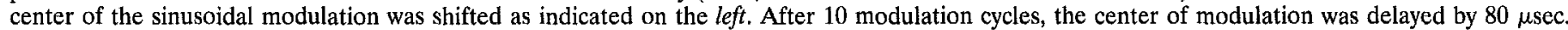

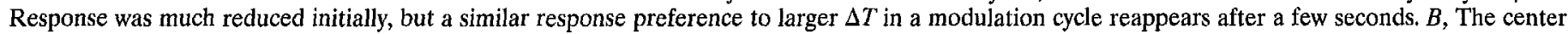

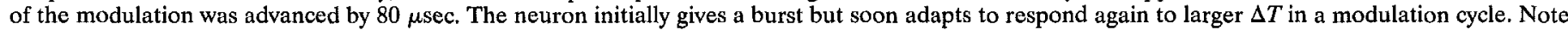

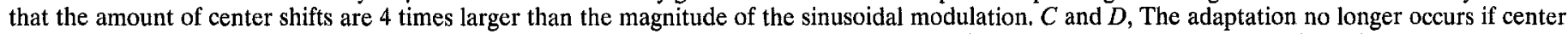

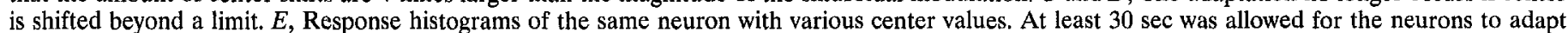

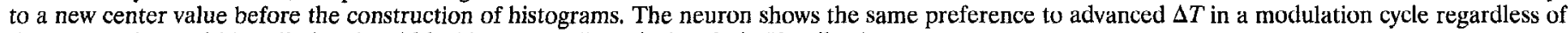
the center phase within a limit. Bin width, $20 \mathrm{msec}$. Full vertical scale is 50 spikes $/ \mathrm{sec}$. 

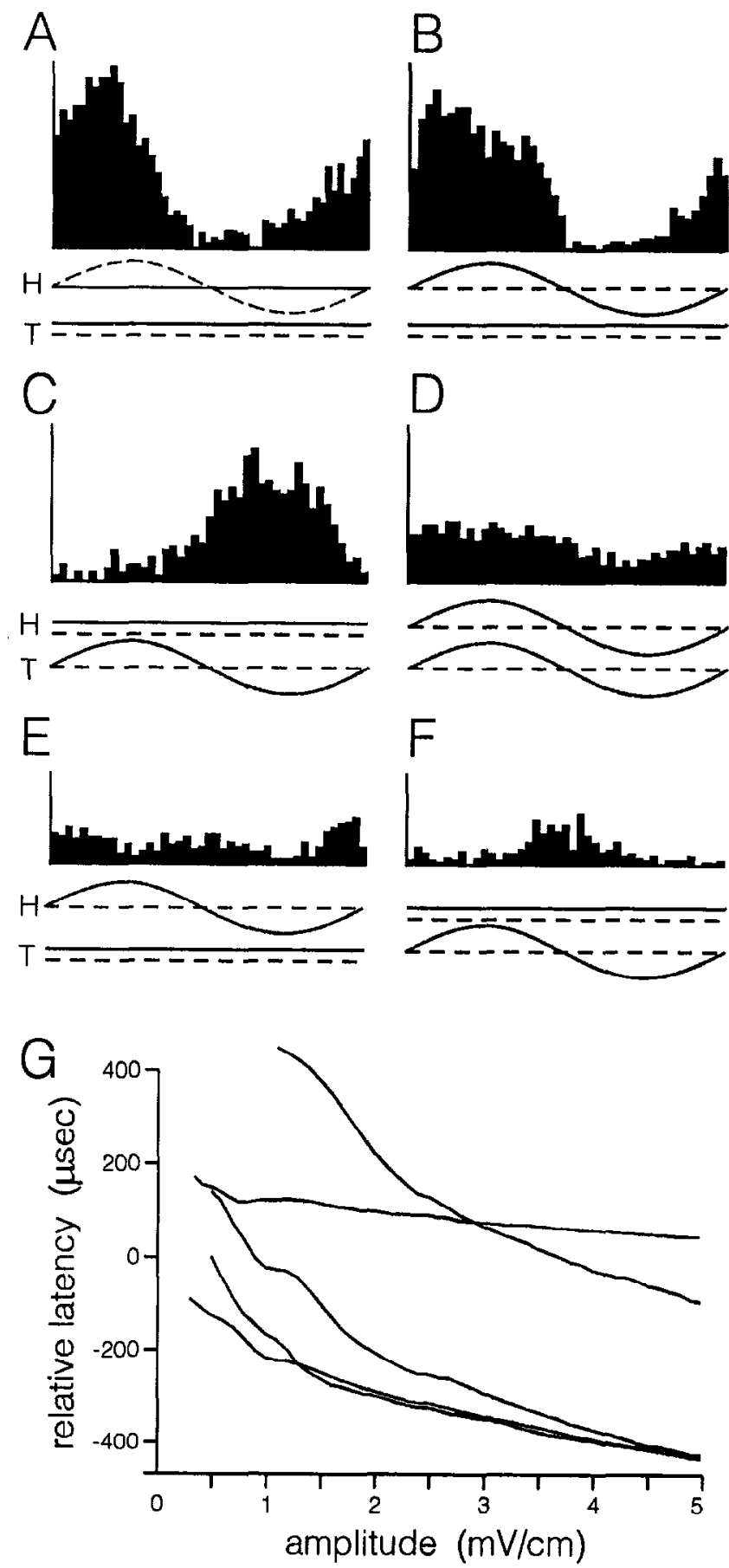

Figure 12. $A-F$, Responses of a differential-phase-sensitive neuron to amplitude modulation. Solid and dotted lines under each histogram represent the modulation of amplitude and absolute phase, respectively, in the stimulus in head $(H)$ and trunk $(T)$ compartments. Modulation magnitude was $\pm 35 \mu \mathrm{sec}$ for phase modulation, \pm 150 $\mu \mathrm{V} / \mathrm{cm}$ for amplitude modulation (mean $2 \mathrm{mV} / \mathrm{cm}$ ) in all cases. $A$, Response profile showing a preference to phase advance of the head stimulus. $B$, Similar response was induced by amplitude modulation in the head without phase modulation in any compartment. $C$, When the identical amplitude modulation was applied to the trunk instead, histogram was shifted by $180^{\circ} . D$, If the identical amplitude modulation was applied to both head and trunk compartments, the response largely disappeared. $E$ and $F$, When phase of carrier signals in the two compartments was placed $180^{\circ}$ out of phase with each other, amplitude modulation of any compartments failed to induce a response. $G$, Latency of firing of intracellularly recorded phase-locked potentials in the ELL. Stimulus amplitude was incremented by $100 \mu \mathrm{V} / \mathrm{cm}$ steps from 0 to $5 \mathrm{mV} / \mathrm{cm}$ in five neurons. that the response profile does not recover completely, but mean firing rate is a function of the center phase. This suggests that a feedback mechanism is involved in the adaptation process. The differential-phase-sensitive neurons found in this study have a large branched apical dendrite (Fig. 11) morphologically similar to that found in amplitude processing neurons in the ELL of gymnotiform electric fishes. Descending inputs from the nucleus praeeminentialis to the dendrites of these amplitude-sensitive ELL neurons in gymnotiform fishes are crucial in mediating adaptation to mean amplitude changes (Bastian and Bratton, 1990; Bastian and Courtright, 1991). A remarkably similar type of adaptation to the steady-state change in phase difference was observed in the torus of Eigenmannia (Rose and Heiligenberg, 1986).

\section{Responses of differential-phase-sensitive neurons to amplitude modulation}

As shown in Figure $12 G$, small amplitude changes within the physiological range in electrosensory signals induced an amplitude-dependent latency shift that is large enough to evoke responses in differential-phase-sensitive neurons; thus, phase and amplitude information is mixed and ambiguously represented. Although the JAR relies on the time course of amplitude and phase modulation (see Fig. 2 in Kawasaki, 1993a), complete separation of amplitude and phase information is not necessary. The direction of frequency shifts of EOD in the JAR is determined by the sense of rotation of a circular graph in the amplitude-phase plane representing sensory stimulus. The crosstalk between phase and amplitude only skews the centrally represented Lissajous circle into a slanted ellipsoid whose sense of rotation still could be determined. Gymnarchus does show the JAR in response to pure amplitude modulation in one compartment without a differential phase modulation. These two points can be explained by an experiment performed in Eigenmannia (see Fig. 13 in Heiligenberg and Bastian, 1980).

Although sensitivity of differential-phase-sensitive neurons to amplitude modulation makes JAR computation more complicated, sensitivity to amplitude allows these neurons to participate simultaneously in electrolocation by encoding amplitude differences between body areas. Amplitude differences could be encoded by neurons that have excitatory and inhibitory amplitudesensitive receptive fields. Although such neurons are found in the ELL of gymnotiform fishes (Bastian, 1986), the experiment shown in Figure 12, $E$ and $F$, demonstrates that the differential-phasesensitive neurons in Gymnarchus are not of this kind and, indeed, do respond to phase differences induced by amplitude differences. Differential-amplitude coding may have been the primary evolutionary function of the differential-phase system that may be used later by the JAR system as a phase-encoding mechanism for the JAR.

\section{Comparative implication}

In an independently evolved electric fish, Eigenmannia, the computation of phase difference is carried out in the torus semicircularis (hereafter "torus") of the midbrain. Phase-locked inputs from electroreceptors in Eigenmannia terminate onto the soma of spherical cells in the ELL. The spherical cells, however, send single axons only to the torus without making any projections within the ELL. Their axons make contacts to adendritic giant 
neurons and the small cells in the torus that perform phase comparison (Heiligenberg and Rose, 1985; Carr et al., 1986a,b).

The phase comparison circuits within the ELL of Gymnarchus and within the torus of Eigenmannia are similarly organized: (1) phase information is supplied to a phase-comparing element (ICL neurons in Gymnarchus, small cells in Eigenmannia) directly from the phase-locked inputs (S-type afferent in Gymnarchus, spherical cell afferents in Eigenmannia), and indirectly via giant neurons; (2) phase comparison occurs at a layered structure (the ICL in Gymnarchus, the lamina-VI in Eigenmannia); and (3) adendritic giant neurons spread large axonal arbors within the structures.

The present finding that Gymnarchus and Eigenmannia perform phase comparison by different brain structures provides further evidence that these species came to possess the JAR by convergent evolution.

Mormyrid electric fishes, which are closely related to Gymnarchus, appear to detect phase information by knollenorgan electroreceptors. Afferents from this type of electroreceptor terminate onto the soma of adendritic neurons in the nucleus of ELL, much as they do in Gymnarchus. Unlike in Gymnarchus, these neurons do not project within the ELL but, rather, send single axons to the torus. In mormyrid electric fishes, phase comparison is presumed to occur in the midbrain (Szabo et al., 1975; Enger et al., 1976; Bell et al., 1981).

These comparisons suggest that different brain structures may perform functionally similar tasks in independently evolved systems that have a similar overall function; conversely, we may speculate that homologous structures are not assigned necessarily a similar function even in closely related species.

\section{REFERENCES}

Bass AH, Hopkins CD (1982) Comparative aspects of brain organization of an African "wave" electric fish, Gymnarchus niloticus. J Morphol $174: 313-334$

Bastian J (1986) Electrolocation. Behavior, anatomy, and physiology. In: Electroreception (Bullock TH, Heiligenberg W, eds), pp 577-612. New York: Wiley.

Bastian J, Bratton B (1990) Descending control of electroreception. I. Properties of nucleus praeeminentialis neurons projecting indirectly to the electrosensory lateral line lobe. J Neurosci 10:1226-1240.

Bastian J, Courtright I (1991) Morphological correlates of pyramidal cell adaptation rate in the electrosensory lateral line lobe of weakly electric fish. J Comp Physiol 168:393-407.

Bastian J, Heiligenberg W (1980) Neural correlates of the jamming avoidance response in Eigenmannia. J Comp Physiol 136:135-152.

Bell CC, Finger TE, Russell CJ (1981) Central connections of the posterior lateral line lobe in mormyrid fish. Exp Brain Res 42:9-22.

Bell CC, Libouban S, Szabo T (1983) Pathways of the electric organ discharge command and its corollary discharges in mormyrid fish. $\mathbf{J}$ Comp Neurol 216:327-338.

Bullock TH, Behrend K, Heiligenberg W (1975) Comparison of the jamming avoidance responses in Gymnotoid and Gymnarchid electric fish: a case of convergent evolution of behavior and its sensory basis. $\mathbf{J}$ Comp Physiol 103:97-121.

Carr CE, Boudreau RF. (1993) Organization of the nucleus magnocellularis and the nucleus laminaris in the barn owl: encoding and measuring interaural time differences. J Comp Neurol 334:337-355.
Carr CE, Heiligenberg W, Rose GJ (1986a) A time-comparison circuit in the electric fish midbrain. I. Behavior and physiology. J Neurosci 6:107 119

Carr CE, Maler L, Taylor B (1986b) A time-comparison circuit in the electric fish inidbrain. II. Functional morphology. J Neurosci 6:13721383.

Enger PS, Libouban S, Szabo T (1976) Rhombo-mesencephalic connections in the fast conducting electrosensory system of the mormyrid fish, Gnathonemus petersii. An HRP study. Neurosci Lett $3: 239-243$

Frank K, Becker MC (1964) Microelectrodes for recording and stimulation. In: Physical techniques in biological research (Nastuk W, ed), pp 23-84. New York: Academic.

Goldberg JM, Brown PB (1969) Response of binaural neurons of dog superior olivary complex to dichotic tonal stimuli: some physiological mechanisms of sound localization. J Neurophysiol 32:613-636.

Harnischfeger G, Neuwiler G, Schlegel P (1985) Interaural time and intensity coding in superior olive complex and inferior colliculus of the echolocating bat, Molossus ater. J Neurophysiol 53:89-109.

Heiligenberg W (1991) Neural nets in electric fish. Cambridge, MA: MIT.

Heiligenberg W, Bastian J (1980) The control of Eigenmannia's pacemaker by distributed evaluation of electroreceptive afferences. J Comp Physiol 136:113-133.

Heiligenberg W, Rose G (1985) Phase and amplitude computations in the midbrain of an electric fish: intracellular studies of neurons participating in the jamming avoidance response of Eigenmannia. $\mathbf{J}$ Ncurosci 5:515-531.

Hopkins CD (1974) Electric communication in fish. Am Sci 62:426-437.

Horikawa K, Armstrong WE (1988) A versatile mean of intracellular labeling: injection of biocytin and its detection with avidin conjugates. $\mathrm{J}$ Neurosci Methods 25:1-12.

Kawasaki M (1993a) Independently evolved jamming avoidance responses employ identical computational algorithms: a behavioral study of the African electric fish, Gymnarchus niloticus. J Comp Physiol 173:9-22.

Kawasaki M (1993b) Temporal hyperacuity in the gymnotiform electric fish, Eigenmannia. Amer Zoo 33:86-93.

Kawasaki M (1994) The African wave-type electric fish, Gymnarchus niloticus, lacks corollary discharge mechanisms for electrosensory gating. J Comp Physiol 174:133-144.

Kawasaki M, Rose GJ, Heiligenberg W (1988) Temporal hyperacuity in single neurons of electric fish. Nature 336:173-176.

Konishi M, Takahashi TT, Wagner H, Sullivan WE, Carr CE (1988) Neurophysiological and anatomical substrates of sound localization in the owl. In: Auditory function (Edelman GM, Gall WE. Cowan WM, eds), pp 721-745. New York: Wiley.

Lauder GV, Liem KF (1983) Patterns of diversity and evolution in rayfinned fishes. In: Fish neurobiology (Northcutt RG, Davis RE, eds), pp 1-24. Ann Arbor: University of Michigan.

Maler L, Sas E, Rogers J (1981) The cytology of the posterior lateral line lobe of high frequency weakly electric fish (Gymnotidae). Dendritic differentiation and synaptic specificity in a simple cortex. J Comp Neurol 195:87-139.

Moiseff A, Konishi M (1981) Neuronal and behavioral sensitivity to binaural time differences in the owl. J Neurosci 1:40-48.

Rose GJ, Heiligenberg W (1986) Limits of phase and amplitude sensitivity in the torus semicircularis of Eigenmannia. J Comp Physiol A 159:813-822

Szabo T, Sakata H, Ravaille M (1975) An electrotonically coupled pathway in the central nervous system of some teleost fish, Gymnotidae and Mormyridae. Brain Res 95:459-474. 